\title{
Laboratory modeling of flow regimes in a draft tube of Francis hydro-turbine
}

\author{
Sergey Shtork ${ }^{1,2^{*}}$, Ivan Litvinov ${ }^{1,2}$, Andrey Mitryakov ${ }^{1,2}$, and Kemal Hanjalic ${ }^{2,3}$ \\ ${ }^{1}$ Institute of Thermophysics SB RAS, 630090 Novosibirsk, Russia \\ ${ }^{2}$ Novosibirsk State University, 630090 Novosibirsk, Russia \\ ${ }^{3}$ Delft University of Technology, Delft, Netherlands
}

\begin{abstract}
The paper reports on some results of the experimental study of flow and pressure pulsations in a laboratory model of the draft tube (DT) of Francis-99 hydro-turbine over a broad range of operating regimes. Velocity distributions at the model inlet varied within 866 modes of the turbine load conditions, including those with maximum coherent pressure pulsations on the model walls. The contact and noncontact methods were used to measure pressure pulsations on the model walls using acoustic sensors and to measure the averaged velocity distribution with a laser Doppler anemometer "LAD-06i". Analysis of the results have showed that in the model cone there are flow modes with forming of precessing vortex cores, accompanied by a sharp increase in the amplitude of coherent pressure pulsations on the wall and the velocity field rearrangement. It is shown that the vortex core starts forming with an increase in the integral parameter of swirl up to $S>0.5$. A novelty of the work is the combination of the rapid prototyping (3-d printing) of the inflow swirl generators and computerized measurement techniques that makes it possible to acquire rapidly a large amount of experimental data for a variety of designs and operating conditions. The results can provide insight into the effect of various design and operating parameters on the flow physics, as well as serve for verification of the numerical simulations.
\end{abstract}

\section{Introduction}

The growing energy consumption nowadays imposes high requirements to the hydroelectric power plants. This is because electricity production must be adjusted, considering ups and downs of the load of electric mains. Varying the generator load results in short-term changes in rotation frequency of the turbine impeller, thereby the flow behind it becomes swirling. This leads to the formation of unsteady flow regimes under which the vortex instability of the swirling flow behind the impeller is formed. It is known as precessing vortex core (PVC) [1-3]. This effect causes strong periodic pressure pulsations that propagate throughout the water column due to the presence of an elbow in the HPP drainage tract (draft tube) [4-6]. The vortex rotation frequency is sufficiently low and lies in the same range with fundamental frequencies of the hydraulic unit structure, which can lead to uncontrolled resonance and vibrations. In addition to the risk of equipment damage and reduced performance, this effect may decrease the turbine efficiency. These facts make the PVC effect an important and urgent problem of hydropower engineering and hydrodynamics.

To date, computer simulation is the main tool for modeling complex three-dimensional unsteady flows in the flow path of the turbine [7,8]. However, numerical methods need to be validated by experimental data.
Since the experiments on full-scale plants are difficult or impossible, the verification of numerical methods is usually realized on a smaller laboratory model of the turbine flow path. For simplifying the model design the experiments may be performed with an air as the working medium instead of water $[9,10]$. Besides, to specify the velocity distribution realized behind a real turbine it is not necessary to model the entire hydraulic path of the hydraulic turbine. Instead, a combination of two swirlers with a special profile of vanes may be used. For example, the work $[11,12]$ presents a method that using a combination of fixed and rotating vane swirlers allows reproducing velocity distributions at the inlet of the draft tube model, which are very close to those behind the full-scale Francis turbine.

To date, the mechanisms of the PVC formation have not been revealed in full detail. A clear relationship between the velocity distribution behind the impeller and the level of pressure pulsations caused by the PVC are not fully understood either. It is in this context, the present work investigates the flow regimes with strong pressure pulsations in the model draft tube of hydraulic turbine, the vortex nature of these pulsations and relation to the PVC effect. Analysis of the velocity distributions allowed identifying indicators of the PVC formation by varying the input parameters of the test stand.

\footnotetext{
*Corresponding author: shtork@itp.nsc.ru
} 


\section{Experimental techniques}

As already mentioned, using a combination of the two vane swirlers it is possible to obtain the velocity distribution close to that, implemented behind a real impeller, while avoiding the need in reproducing the entire turbine path. This approach not only greatly simplifies and cheapens the experimental rig construction, but also provides additional opportunities to expand the range of input conditions in the draft tube. Among other things, it enables obtaining and investigating the modes, which cannot yet be implemented on existing designs of turbines, but they are promising in terms of reducing level of pulsations and improving energy efficiency.

In these experiments, we used a pair of swirlers, whose geometry is presented in [13-14]. The blades were designed for optimum performance of Francis hydraulic turbines and calculated for volumetric flow rate $Q_{c}=$ $48.5 \mathrm{l} / \mathrm{s}$ and rotation frequency of the runner $n_{c}=40.5$ Hz. A set of swirlers was made by rapid prototyping using a 3D-printer Cube X Duo. As the draft tube design for Francis turbines we chose the model geometry Francis-99, which is widely used to validate numerical calculations $[15,16]$.

The experiments were carried out on an open aerodynamic setup with the test section, produced by the method of rapid prototyping (Fig. 1). The air flow was generated by a blower with a rated flow rate and pressure $Q_{\max }=550 \mathrm{~m}^{3} / \mathrm{h}, P=0.4 \mathrm{bar}$, respectively. The airflow rate in the wind tunnel circuit was regulated using a frequency converter and measured by an ultrasonic flowmeter. Air flow was supplied to the flow path of the working area over six axisymmetric nozzles and passed through a couple of honeycombs; then the flow was further leveled using a profiled nozzle. The initial swirl was imparted to the flow at a stationary swirler (guide vane); then the rotating swirler (impeller) redistributed the swirl, thereby simulating the desired velocity distribution at the inlet to the draft tube. The servomotor drove the impeller into rotation with a frequency in the range from 0 to $50 \mathrm{~Hz}$. Operating parameters, flow rate $Q$ and rotation frequency $n$ were set with the accuracy of 1.5 and $0.5 \%$, respectively, using original software, providing the feedback control.

To register pressure pulsations we used acoustic sensors based on microphones Behringer ECM 8000, whose signals were digitized by the ADC L-Card E-440. Profiles of the averaged velocity and RMS velocity fluctuations of the flow were measured using the laser Doppler anemometer "LAD-06i". As tracers we used particles of paraffin oil aerosol, produced by Laskin atomizer, which allows obtaining droplets with a characteristic size of 1-3 $\mu \mathrm{m}$ [17].

\section{Results}

The initial stage of the work was determining the modes with maximum pressure pulsations that appear in a draft tube (DT) cone at deviating from the design mode $\left(Q_{c}\right.$ and $\left.n_{c}\right)$ due to the generation of the precessing vortex core. The calculated parameters corresponding to the optimal mode of the turbine operation were taken from [13-14]. In the experiments, two acoustic sensors were placed in the middle of the cone from opposite sides flush with the cone walls (Fig. 1). Operating parameters of the setup ranged for the flow rate from $0.39 Q_{c}$ to 1.23 $Q_{c}$, and for the frequency of the impeller rotation from $0.2 n_{c}$ to $1.23 n_{c}$. The signal from the sensors was recorded for 10 seconds with a sampling frequency of 2 $\mathrm{kHz}$.

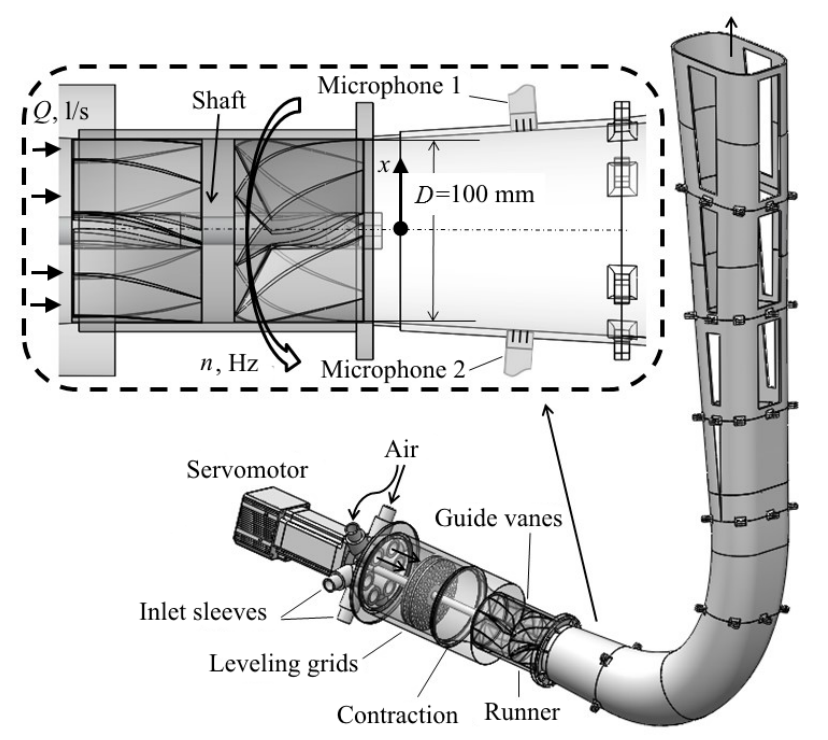

Fig. 1. Scheme of experimental setup (the insert shows location of the measuring microphones and the measuring section $I$, spaced from the rear edge of the central body to $4 \mathrm{~mm}$; $D$ is the inlet diameter of the conical part of the draft tube).

Fig. 2 presents a diagram based on the measuring results for the 866 operation modes of the unit corresponding to nonoptimal conditions. The diagram shows maximum amplitudes in the spectra of the differential signal of two sensors depending on the flow rate and rotation frequency of the impeller. The differential signal was used to enhance the contribution from the precessing vortex and suppress the in-phase component of pulsations, whose source was noises of the aerodynamic contour. It may be seen that in the upper left part of the diagram there is the region corresponding to high levels of pressure pulsations.

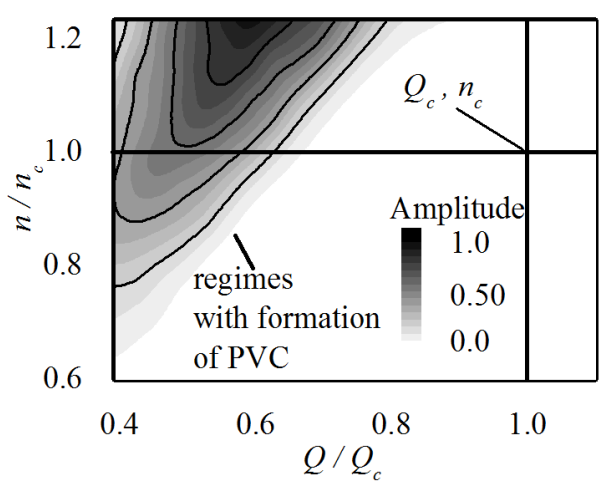

Fig. 2. The dependence of pulsations on the operating parameters $Q$ and $n$. The scale is given in arbitrary units. 


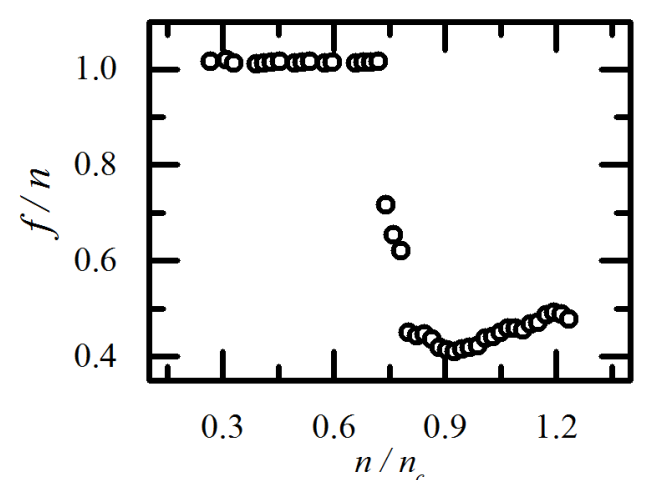

Fig. 3. The dependence of the maximum amplitude frequency in the spectrum of the difference signal of two sensors on rotation frequency of the swirler.

These pulsations are most likely caused by the precessing vortex core, which is formed in the cone of the draft tube at nonoptimal operation modes. Fig. 3 presents the dependence of the maximum amplitude frequency in the spectrum of the difference signal of the microphones upon the impeller frequency at a fixed flow rate $0.59 Q_{c}$. According to this figure, up to a frequency of $0.75 n_{c}$ in the spectra there is a dominating peak corresponding to the frequency of the swirler rotation $n$. Then, starting from the rotation frequency $0.8 n_{c}$ in the spectrum begins to dominate the frequency of about 0.42 $n$, associated with the development of vortex instability in the form of PVC. With further increase in the rotation frequency, the dominant frequency slowly grows.

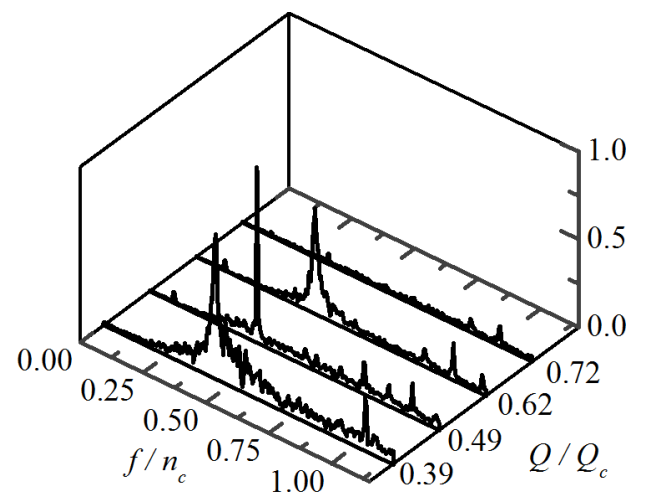

Fig. 4. Waterfall diagram of the spectra of the difference signal of two acoustic sensors. The vertical axis is given in relative units.

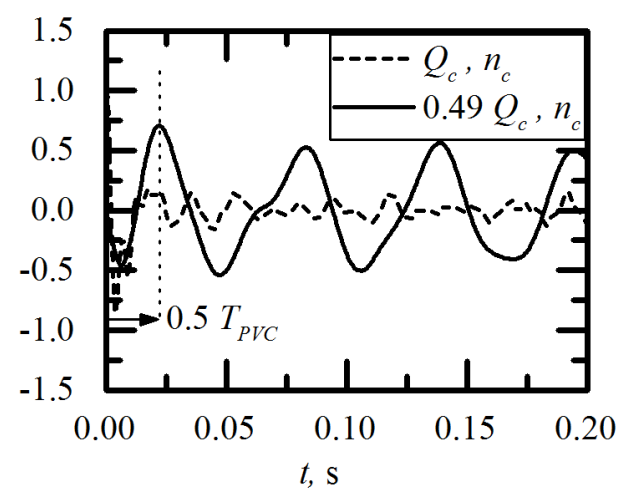

Fig. 5. Cross-correlation functions for different modes of $Q$ (relative units are plotted on y-axis).
Fig. 4 shows evolution of the spectra of the differential signal for different flow rates at the fixed rotation frequency $n_{c}$. It is seen that the dominant peak in the spectra, which is associated with the rotation frequency of the precessing vortex, first increases in its level, and starting from the flow rate $0.39 Q_{c}$ reaches maximum at the flow rate of $0.49 Q_{c}$, and then at $0.79 Q_{c}$ disappears completely from the spectrum.

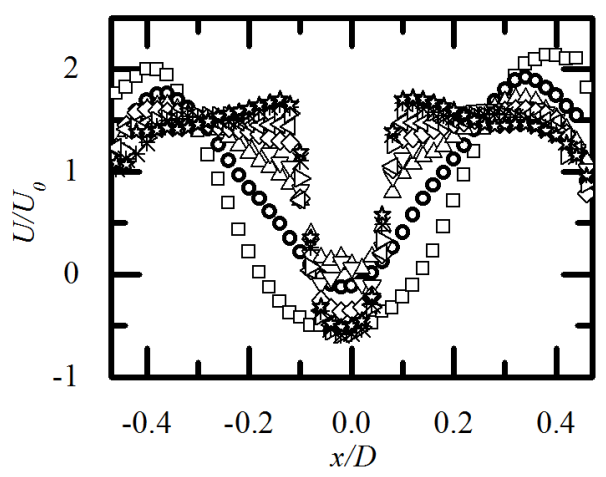

(a)

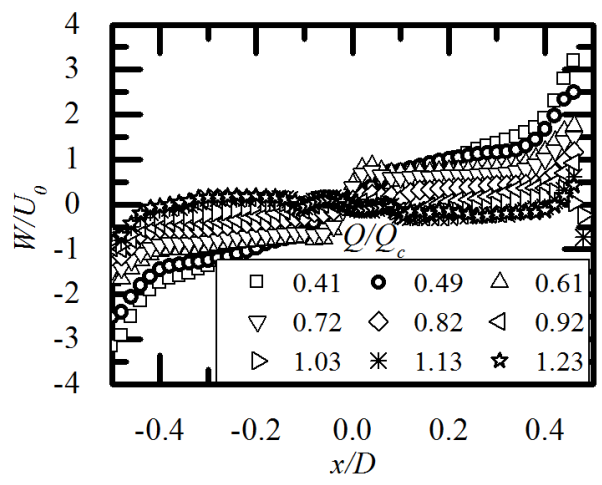

(b)

Fig. 6. Profiles of the averaged velocity: axial (a), tangential component (b).

To confirm that the precessing vortex motion gives the largest contribution to pressure pulsations at nonoptimal modes Fig. 5 shows the cross-correlation functions, built for two regimes (optimal and nonoptimal, with maximum pulsations) with flow rates $Q_{c}$ and $0.49 Q_{c}$, respectively, at a constant rotation frequency of the swirler equal to $n_{c}$. It is seen that for the optimal mode the maximum cross-correlation function caused by the noise is only present in zero, and no periodicity of the cross-correlation function is observed. For a signal corresponding to the non-optimal regime, the maximum of the function falls on $1 / 2$ of the PVC period. This is because the sensors detect the pressure disturbance in the opposite phase. I.e. one sensor registers the pressure pulse caused by the vortex passage, and then, in a half period of the PVC precession, the pulse is registered by the second sensor. Thus, in the cone the vortex loses its axial position and executes a precessional movement, coming alternately to one and then to another sensor.

To analyze the velocity profiles, of the whole set of investigated operating conditions we selected the flow rates corresponding to $0.41,0.49,0.61,0.72,0.82,0.92$, $1.03,1.13$ and $1.23 Q_{c}$, at a fixed rotation frequency of the swirler equal to $n_{c}$. The averaged distributions of 
axial and tangential velocity components were measured in the control section, located at a distance of $4 \mathrm{~mm}$ behind the bluff body. Fig. 6a presents profiles of averaged axial velocity. For profiles, corresponding to 0.41 and $49 Q_{c}$, a wide area of the reverse flow and the parabolic shape of the profile may be seen. They serve as a good indicator of the PVC formation [18-19]. With further increase in the flow rate the reverse flow area is reduced to the size of the bluff body $-0.2 D$ ( $D$ is the diameter of the inlet orifice of the DT cone). Distribution of tangential velocity is characterized by a decrease in the level of maximum velocity (Fig. 6b). For the modes with 1.13 and $1.23 Q_{c}$ the regions with the opposite sign of swirl are observed.

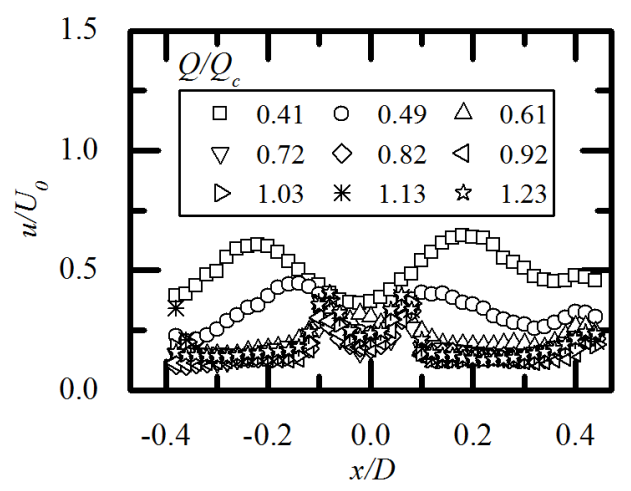

(a)

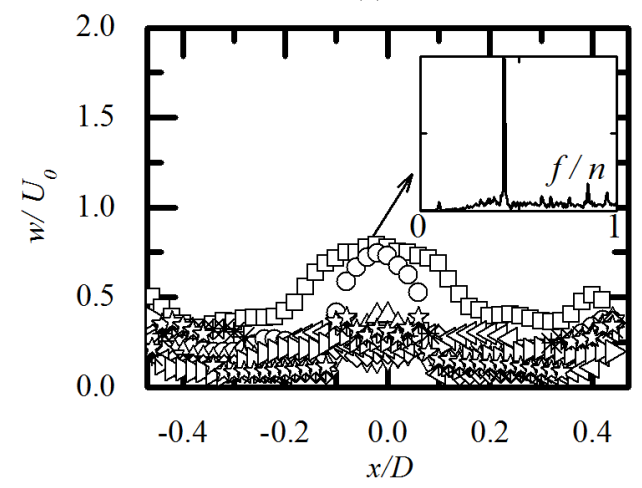

(b)

Fig. 7. Profiles of the RMS averaged velocity: axial (a), tangential components (in the inset there is Lomb spectrum for the mode with $0.49 Q_{c}, n_{c}$ ) (b).

Characteristic profiles of mean square deviations (RMS) of axial and tangential velocity components are presented in Fig. 7 for the flow rate $0.82 Q_{c}$ and the rotation frequency $\mathrm{n}=n_{c}$. Distributions of axial velocity pulsations show that at low flow rate the level of pulsations reaches $0.5 U_{0}$, and at the flow rate over 0.61 $Q_{c}$ it falls to $0.2 U_{0}$. Moreover, the positions of the maxima of the axial velocity RMS change from $0.2 D$ to $0.1 D$, which is associated with the flow rearrangement and the PVC effect suppression due to reduction in the radius of the vortex precession. RMS of tangential velocity shows that the pulsations change from $0.63 U_{0}$ to $0.25 U_{0}$ at increasing flow rate, and the position of the maximum tangential velocity is shifted to the axis of flow rotation.

To prove the vortex nature of the pulsations we present the spectrum of pulsations of the tangential velocity component in the vicinity of the cone center. To build the spectrum of non-equidistant velocity signal use the Lomb algorithm [20]. Insert in Fig. 7b shows the relative amplitude of LS - the Lomb spectrum of pulsations of tangential velocity component for the flow rate of $0.49 Q_{c}$ and rotation frequency $n_{c}$. The presence of a narrow peak at the PVC frequency correlates with the corresponding spectrum of the differential signal, which shows the vortex nature of the pulsations.

Non-optimal flow regimes behind the hydro-turbine, distinguished by a strong residual swirl, can be characterized by the swirl parameter:

$$
S=\frac{\int_{\Sigma} W U r d \Sigma}{(D / 2) \int_{\Sigma} U^{2} d \Sigma},
$$

where $U$ is the axial velocity component, $W$ is the tangential velocity component, $d \Sigma$ is the element of area, and $D$ is the inlet diameter of the draft tube cone.

Fig. 8 shows the dependence of the integral parameter of the swirl, calculated from the averaged velocity profiles (Fig. 6).

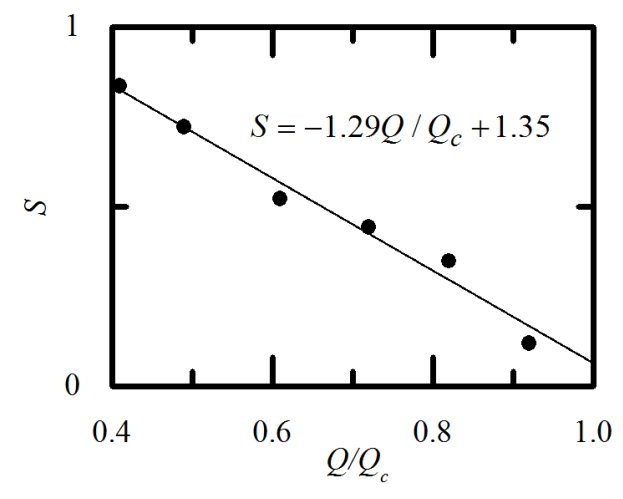

Fig. 8. The dependence of the integral parameter of the swirl on the flow rate at a fixed rotation frequency $n_{c}$.

The dependence with good accuracy represents a linear function $\mathrm{S}=1.29 Q / Q_{c}+1.35$. It becomes zero at the flow rate $1.04 Q_{c}$, which is very close to optimal conditions, and the maximum value $S=0.83$ is obtained for $Q=0.41 Q_{c}$. The formation of a vortex core is observed for $\mathrm{S}>0.5$, which corresponds to the critical value known from the literature, after which the vortex breakdown, the formation of the reverse flow zone and the PVC generation begin [1].

Thus, the paper illustrates the usefulness of the rapid prototyping of physical models for experimental study of flow unsteadiness and emergence of the non-stationary vortex structures for a variety of swirler designs and a range of operating conditions. Such results can provide insight into the effect of various design and operating parameters on the flow physics, as well as serve for verification of the numerical simulations. Despite the neglect of cavitation and possible scaling effects, the approach can be regarded as useful for fast gathering of information at much reduced expenses compared to large-scale experiments or massive numerical simulations. 


\section{Conclusions}

The work has shown that the deviation from the optimal calculated regime to the area, corresponding to the modes of real turbine underload, results in generating the vortex instability in the form of the PVC. The presence of precession motion of the vortex and its relationship with the regime of maximum pressure pulsations in the draft tube cone have been demonstrated through the evolution of spectra and analysis of crosscorrelation functions of the pressure pulsations.

The analysis of the measured profiles of the averaged axial velocity component has revealed a wide area of the reverse flow of the parabolic shape for the modes with PVC formation. When approaching the optimal regime (increasing flow rate) the central valley on the axial velocity profiles is narrowed to the transverse dimension of the bluff body. The tangential velocity component in the vicinity of the optimal regime tends to zero value. Pulsation spectra of the tangential velocity confirm the PVC formation with precession frequency of $0.4-0.42 n_{c}$ for low flow rates 0.41 and $0.49 Q_{c}$, corresponding to non-optimal regimes. The swirl parameter calculated on velocity profiles for different flow rates is a linear dependence on the flow rate. At that the increased level of pulsations, caused by the PVC, and the central area of the reverse flow appear at $S>0.5$, which corresponds to the critical value known from the literature. If this level is exceeded in a swirling flow the breakdown of the vortex and the generation of the PVC take place.

The study was performed with financial support of the Russian Science Foundation (project No. 14-29-00203).

\section{References}

1. A. Gupta, D. Lilley, and N. Syred, Swirl flows (Abacus Press, London, 1984)

2. E. Fernandes, M. Heitor, and S. Shtork, Exp. Fluids, 40 2006

3. I. Litvinov, D. Sharaborin, S. Shtork, Thermophys. Aeromech., 22 (2015)

4. P. Deriaz, $1^{\text {st }}$ Symposium IAHR, (1960)

5. O. Kirschner, A. Ruprecht, and S. Riedelbauch, $3^{\text {rd }}$ Symposium IAHR, (2009)

6. B. Mulu, P. Jonsson, and M. Cervantes, Appl. Energy, 93 (2012)

7. A. Minakov, D. Platonov, A. Dekterev, A. Sentyabov, and A. Zakharov, Int. J. Heat Fluid Flow, 53 (2015)

8. Z. Zuo, S. Liu, D. Liu, D. Qin, and Y. Wu, J. Hydr. B, 27 (2015)

9. M. Nishi, K. Yoshida, M. Yano, M. Okamoru, K. Miyagawa, and S. Liu, $2^{\text {nd }}$ Symposium IAHR, (2007)

10. N. Povh, Aerodynamic experiment in machinery (Leningrad, 1974, in Russian)

11. R. Susan-Resiga, S. Muntean, and A. Bosioc, The $4^{\text {th }}$ International Symposium on Fluid Machinery and Fluid Engineering, (2008)

12. S. Skripkin, M. Tsoy, S. Shtork, and K. Hanjalić, J. Hydraul. Res., 54 (2016)

13. P. Kuibin, I. Litvinov, V. Sonin, A. Ustimenko, and S. Shtork, Sc. Bull. Novosibirsk State University, 11 (2016), (in Russian)
14. I. Litvinov, A. Mitryakov, and S. Shtork, Power Tech. Eng., 50 (2016)

15. M. Cervantes, C. Trivedi, O. Dahlhaug, and T. Nielsen, J. Phys., 579 (2015)

16. A. Gavrilov, A. Sentyabov, A. Dekterev, adn K.Hanjalić, Int. J. Heat Fluid Fl., (2016), (available online).

17. C. Tropea, A. Yarin, and J. Foss, Springer Handbook of Experimental Fluid Mechanics (Springer-Verlag, Berlin Heideberg, 2007)

18. P. Yazbadabi, Griffiths A., and N. Syred, Exp. Fluids, 17 (1994)

19. I. Litvinov, S. Shtork, P. Kuibin, S. Alekseenko, and K. Hanjalić, Int. J. Heat Fluid Fl., 42 (2013)

20. V. Vityazev, Analysis of irregular time series (S. Petersburg university, 2001, in Russian) 